\title{
A Novel Strategy to Effectively Characterize FinFET Device by Multidirectional Comprehensive Analytical TEM in Semiconductor Wafer-foundries
}

\author{
Wayne Zhao, Bianzhu Fu, Yong Wei, and Irene Brooks \\ Physical Failure Analysis, Center for Complex Analysis, Characterization Group, Fab8, \\ GLOBALFOUNDRIES, Malta, New York, USA.
}

Seeking improved accuracy in an enhanced timely fashion is a constant task facing all physical failure analysis (PFA) and transmission electron microscopy (TEM) teams at wafer-foundries [1 3]. For FinFET semiconductor devices of $14 \mathrm{~nm}$ and beyond, the intrinsic complexity of 3D-FinFET structures becomes escalated. Inevitably, some features at a nanometer scale are thinner than a thickness of a TEM lamella (e.g., around 50 100nm typically, depending on the orientation). This adds to additional complications in straightforward interpretation of the TEM images and sometimes even puzzles integration teams for quick root-cause identification of major detractors impacting wafer yield.

Quick, effective, comprehensive, and straightforward are the buzz words to illustrate the increasingly demanding challenge for PFA support to the business success of wafer foundries. This article is to record a new milestone in our constant and tireless efforts in exploring and implementing novel Analytical TEM approach to comprehensively characterize FinFET transistors of 14nm and beyond. Following our previous publication in 2014 Microcopy \& Microanalysis, which implemented a best practice of converting a planar-view TEM lamella into a new cross-sectional TEM foil in order to provide a quick 3D Analytical TEM visualization of 3-Dimentional FinFET transistors [1], several novel analytical TEM techniques have become more and more mature for daily routine applications, such as STEM tomography. With improved hardware and software, a standard STEM tomography analysis, operated by an expert, can be as quick as 2 hours, which is almost equivalent to the amount of time that an FIB expert needs to extract a thin slice of new TEM lamella from the existing planar-view TEM foil. This motivated us and technically enabled us to improve standard operation protocols (SOP), and to pinpoint true defects more precisely, with a substantial reduction of turnaround time.

The revolution of the upgrade tactic versus our previous approach is to introduce STEM tomography into the analysis loop. Specifically, insert a STEM tomography step into the workflow, right after the regular Analytical TEM on the planar-view TEM sample. If after tomography, a conclusion on rootcause can already be drawn, subsequent steps to physically extract a cross-section from the planar-view may be skipped. If more ambiguity justifies the necessity to continue converting to a cross-section TEM foil, the STEM tomography result will help to navigate clearly to the true abnormal location, and thus, enhances the chance to pin-point the defect successfully. Demonstrated here is one successful example for the effectiveness of this novel best practice. Our case study was done for a PFA on a FinFET memory device. Figures 1a 1d were Analytical TEM results from the initial planar-view TEM lamella. Figures-2a was one of prospective visualization of the defect (arrowed) in Figs-2b 2c, postreconstruction of the STEM tomography on the defect region. Figures 3a 3f were the Analytical TEM results from additional TEM foil extracted from the existing planar-view TEM lamella in Fig-1b, navigated by the aid of STEM tomography. The defect appeared as something blocking the sufficient Si removal during Fin-separation process. Given the complexity of the defect, certain details could have been overlooked, if not scrutinized by the new strategy of comprehensive characterization with multidirectional visualization. 
References:

[1] W. Zhao, et al., Microscopy \& Microanalysis, Vol. 20 (Supplement 3), (2014), pp.362 363.

[2] W. Zhao, et al., Proc. $38^{\text {th }}$ International Symposium for Testing and Failure Analysis, (2012), pp. 347 355.

[3] W. Zhao, Symp. Proc. the Material Research Society, 2002 Fall Meeting, (2002), Vol. 738, pp. G7.15.1 6.

[4] Authors would like to express sincere thanks to Nicolas LaManque, Lowell Hodgkins, and Randy Newkirk, for their excellence in TEM-preparation, Yue Zhang for screening the TEM samples, and GLOBALFOUNDRIES Fab8 electrical failure analysis team for the fault-isolation. Thanks also go to Fab8 Characterization Management and Legal team for supports in the publication clearance, and to Ed Crawford for the proof-reading.
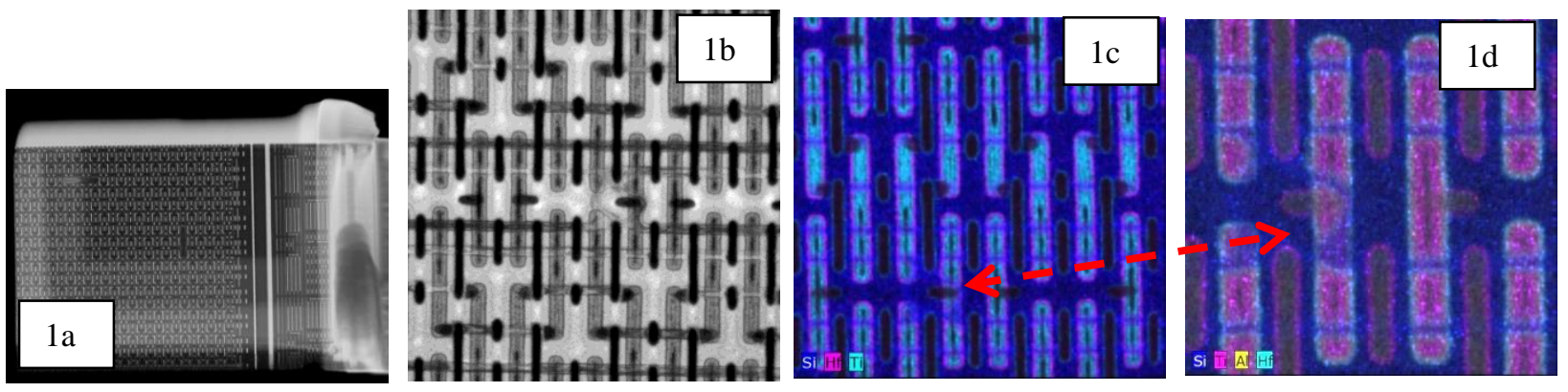

Figure 1. (a) Planar-view TEM; (b) TEM for the defect from (a); (c) and (d) overlay of EDS maps
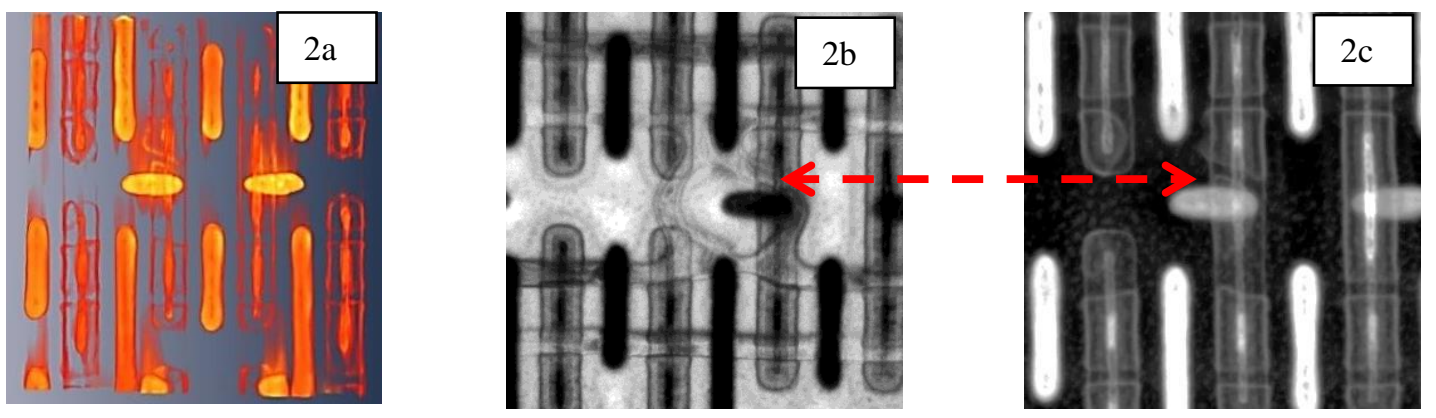

Figure 2. (a) STEM tomography reconstruction for the defect in planar-view TEM foil (b) and (c).
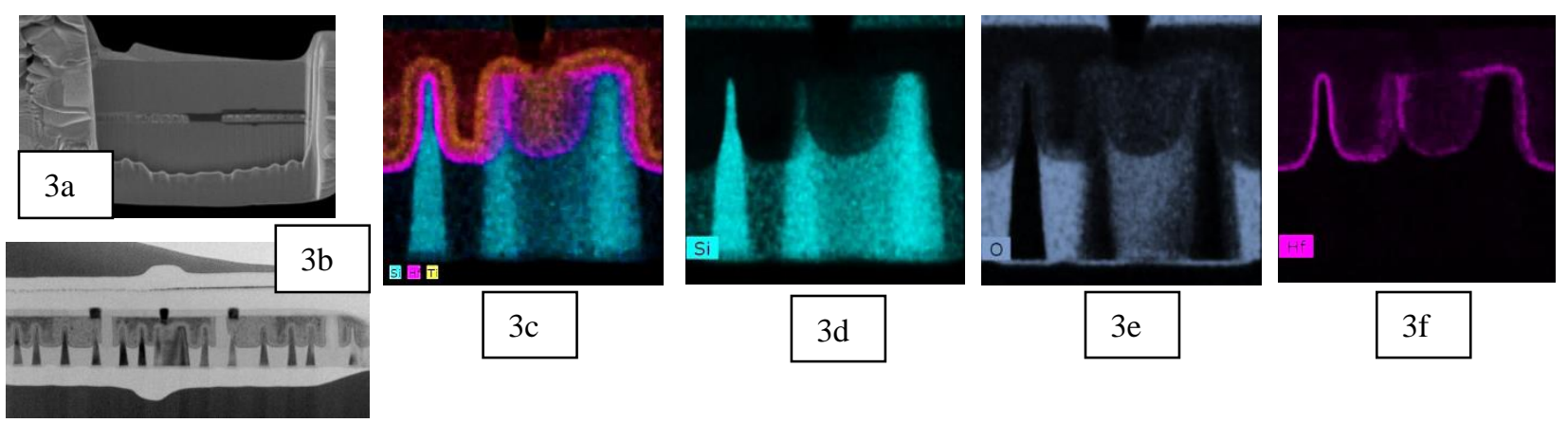

Figure 3. The cross-sectional TEM foil extracted from the planar TEM lamella in Fig-1a, (a) FIB; (b) TEM; (c) overlay of XEDS maps; and (d) (f), individual XEDS maps for Si, O, and Hf, respectively. 